\title{
BROWSER'S NOTES
}

\section{Browser's notes}

Published online: 31 July 2018

(C) ISS 2018

The effect of previous methylphenidate use on incidence of stress fractures in military recruits: a retrospective cohort.

Ben-Ami IS, et al.

J Bone Joint Surg Am. (2018); 100(11):930-5

Patients who have used methylphenidate, a stimulant medication for treatment of attention deficit hyperactivity disorder (ADHD), have been reported to be at greater risk for stress fractures, but lower risk for traumatic fracture. This large, retrospective cohort study sought to explore these findings and reviewed medical records of 100,000 randomly selected combat soldiers who completed compulsory military service in the Israeli Defense Forces. 1451 (1.5\%) subjects carried a diagnosis of ADHD, 689 (47\%) were prescribed stimulant medication and 762 (53\%) were not treated. Diagnosis of fracture, anemia and demographics were compared for these 2 groups and the 98,549 control subjects without a diagnosis of ADHD. Similar age $(\sim 18$ years $)$, height $(\sim 174 \mathrm{~cm})$ and weight $(\sim 67 \mathrm{~kg})$ were found for all groups. The treated ADHD groups had nearly double the percentage of women (13.9\%) compared to the untreated ADHD group (7.5\%) and 2.3 times greater than the control group (5.9\%). Diagnosed anemia was more common in the treated ADHD (9.4\%) and untreated ADHD (8.3\%) groups compared to controls (4.2\%). Stress fractures were more common in the treated ADHD group $(18.3 \%)$ than the control $(13.3 \%)$ group, $p<0.001$. However, no statistical difference was found between the treated ADHD and untreated ADHD (14.6\%) $p=0.07$, or untreated ADHD and controls $(p=0.3)$. The service duration adjusted incidence of traumatic fractures was found to be significantly greater, compared to control, for the treated ( $p=$ 0.01 ) and untreated ADHD group ( $p<0.001)$ groups; but, no difference was found between the ADHD groups. Multivariate logistic regression analysis found treated ADHD was an independent risk factor for both fracture types with adjusted odds ratios (O.R.) of 1.04 and 1.03 for stress and traumatic fractures, respectively. Untreated ADHD was not a risk fracture for stress fractures but was a risk for traumatic fractures $(\mathrm{O} . \mathrm{R} .=1.04)$. Women had a higher risk of stress fracture $(\mathrm{O} . \mathrm{R} .=1.06)$ and men a higher risk for traumatic fracture $(\mathrm{O} . \mathrm{R} .=1.04)$. Anemia increased the risk for stress $($ O.R. = 1.05), but not traumatic fracture. While the study design does not allow a conclusion of causality, ADHD treatment is associated with a slightly higher risk of stress, but not traumatic, fractures. Bone mineral density was not reported.

What is the prevalence of imaging-defined intra-articular hip pathologies in people with and without pain? a systematic review and meta-analysis.

Heerey JJ, et al.

Br J Sports Med. (2018); 52(9):581-93

This analysis included published studies of patients with and without hip, groin and buttock pain, with MR and/or MRarthrography and/or CT imaging, and that also had an outcome measure to determine the prevalence of intraarticular derangement. 29 articles met inclusion criteria and reported on 4410 hips in 2573 subjects; not all studies reported on every articular structure. Labral tears were found in $62 \%$ of symptomatic subjects and $54 \%$ of asymptomatic subjects. Cartilage defects were present in $64 \%$ of symptomatic subjects, but only $12 \%$ of asymptomatic subjects. Edema-like bone marrow lesions were more frequent in the symptomatic group and in subjects with cartilage defects. Synovial herniation pits were found in a similar proportion of symptomatic and asymptomatic patients. Interestingly, MR studies had a higher proportion of pits in symptomatic patients while CT studies reported greater numbers in asymptomatic subjects. The prevalence of paralabral cysts was independent of symptoms. The authors conclude that even though imaging shows a higher prevalence of labral tears and cartilage defects in symptomatic patients, imaging studies must be correlated carefully with clinical signs and symptoms before surgical intervention is undertaken.

\section{Abstracted by C. S. Winalski, M.D. October 2018}

